\title{
Nonlinear Observer Design of the Generalized Rössler Hyperchaotic Systems via DIL Methodology
}

\author{
Yeong-Jeu Sun \\ Department of Electrical Engineering, I-Shou University, Kaohsiung 840, Taiwan \\ Correspondence should be addressed to Yeong-Jeu Sun, yjsun@isu.edu.tw
}

Received 29 February 2012; Accepted 13 April 2012

Academic Editor: Jun-Juh Yan

Copyright (c) 2012 Yeong-Jeu Sun. This is an open access article distributed under the Creative Commons Attribution License, which permits unrestricted use, distribution, and reproduction in any medium, provided the original work is properly cited.

The generalized Rössler hyperchaotic systems are presented, and the state observation problem of such systems is investigated. Based on the differential inequality with Lyapunov methodology (DIL methodology), a nonlinear observer design for the generalized Rössler hyperchaotic systems is developed to guarantee the global exponential stability of the resulting error system. Meanwhile, the guaranteed exponential decay rate can be accurately estimated. Finally, numerical simulations are provided to illustrate the feasibility and effectiveness of proposed approach.

\section{Introduction}

In recent decades, several kinds of chaotic systems have been widely explored; see, for instance, [1-11] and the references therein. This is due to theoretical interests as well as to an efficient tool for chaos synchronization and chaos control design. As a rule, chaos in many systems is a source of the generation of oscillation and a source of instability. Chaotic systems frequently exist in various fields of application, such as system identification, master-slave chaotic systems, secure communication, and ecological systems.

Form practical considerations, it is either impossible or inappropriate to measure all the elements of the state vector. The state observer has come to take its pride of place in system identification, filter theory, and control design. As we know, the tasks of observerbased control systems (with or without chaos) can be divided into two categories: tracking (or synchronization) and observer-based stabilization (or regulation). The state observer can be skillfully applied in observer-based stabilization, synchronization of master-slave chaotic systems, and secure communication. For more detailed knowledge, one can refer to $[1,2,7-$ $9,11-14]$. However, the state observer design of dynamic systems with chaos is in general not as easy as that without chaos. Motivated by the above reasons, the observer design of chaotic 
systems is actually crucial and meaningful. On the other hand, a variety of methods have been proposed for the observer design of systems, such as Chebyshev neural network (CNN), sliding-mode approach, passivation of error dynamics, separation principle, and frequency domain analysis; see, for instance, [15-20] and the references therein.

In this paper, the nonlinear state reconstructor of the generalized Rössler hyperchaotic systems is investigated. Using the DIL methodology, a nonlinear observer for such systems is provided to guarantee the global exponential stability of the resulting error system. Furthermore, the guaranteed exponential decay rate can be correctly estimated. Finally, numerical simulations are given to verify the effectiveness of proposed approach.

\section{Problem Formulation and Main Result}

In this paper, we consider the generalized Rössler hyperchaotic systems as follows:

$$
\begin{gathered}
\dot{x}_{1}(t)=\alpha_{1} x_{1}+\alpha_{2} x_{2}+g_{1}\left(x_{3}, x_{4}\right), \\
\dot{x}_{2}(t)=\alpha_{3} x_{1}+\alpha_{4} x_{2}+g_{2}\left(x_{4}\right), \\
\dot{x}_{3}(t)=r_{1} x_{1} x_{3}+g_{3}\left(x_{4}\right), \\
\dot{x}_{4}(t)=r_{2} x_{3}+g_{4}\left(x_{4}\right), \\
y(t)=x_{4}
\end{gathered}
$$

where $x(t):=\left[\begin{array}{llll}x_{1}(t) & x_{2}(t) & x_{3}(t) & x_{4}(t)\end{array}\right]^{T} \in \mathfrak{R}^{4}$ is the state vector, $y(t) \in \mathfrak{R}$ is the system output, $r_{1}, r_{2}$, and $\alpha_{i}$, for all $i \in\{1,2,3,4\}$ are the system parameters with $r_{1} r_{2} \neq 0$. For the existence and uniqueness of system (2.1), we assume that all the functions $g_{i}(\cdot)$, for all $i \in$ $\{1,2,3,4\}$, are sufficiently smooth.

The following assumption is made on system (2.1) throughout this paper.

(A1) There exists a constant $h_{1}$ such that

$$
h_{1}>\alpha_{4}, \quad h_{1} \alpha_{4}<-\alpha_{2} \alpha_{3} .
$$

Remark 2.1. It is noted that the Rössler hyperchaotic system [21] is the special cases of system (2.1) with

$$
\begin{aligned}
& \alpha_{1}=0, \quad \alpha_{2}=-1, \quad \alpha_{3}=1, \quad \alpha_{4}=0.25, \quad r_{1}=1, \quad r_{2}=-0.5, \\
& g_{1}\left(x_{3}, x_{4}\right)=-x_{3}, \quad g_{2}\left(x_{4}\right)=x_{4}, \quad g_{3}\left(x_{4}\right)=3, \quad g_{4}\left(x_{4}\right)=0.05 x_{4} .
\end{aligned}
$$

The objective of this paper is to search a nonlinear observer for system (2.1) such that the global exponential stability of the resulting error systems can be guaranteed. Before presenting the main result, let us introduce a definition which will be used in the main theorem. 
Definition 2.2. System (2.1) is exponentially state reconstructible if there exist an observer $E \dot{\hat{x}}(t)=g(\widehat{x}(t), y(t))$ and positive numbers $k$ and $\alpha$ such that

$$
\|e(t)\|:=\|x(t)-\widehat{x}(t)\| \leq k \exp (-\alpha t), \quad \forall t \geq 0,
$$

where $\widehat{x}(t)$ expresses the reconstructed state of system (2.1). In this case, the positive number $\alpha$ is called the exponential decay rate.

Now we present the main result for the state observer of system (2.1).

Theorem 2.3. System (2.1) with (A1) is exponentially state reconstructible. Besides, a suitable nonlinear observer is given by

$$
\begin{gathered}
r_{1} \widehat{x}_{3} \dot{\hat{x}}_{1}=\left(\alpha_{1}+h_{1}\right)\left[\dot{\hat{x}}_{3}-g_{3}(y)\right]-h_{1} r_{1} \widehat{x}_{1} \widehat{x}_{3}+r_{1} \widehat{x}_{3}\left[\alpha_{2} \widehat{x}_{2}+g_{1}\left(\widehat{x}_{3}, y\right)\right], \\
\dot{\hat{x}}_{2}=\alpha_{3} \widehat{x}_{1}+\alpha_{4} \widehat{x}_{2}+g_{2}(y), \\
\widehat{x}_{3}=\frac{1}{r_{2}}\left[\dot{y}-g_{4}(y)\right], \\
\widehat{x}_{4}=y .
\end{gathered}
$$

In this case, the guaranteed exponential decay rate is given by $\alpha:=1 / \lambda_{\max }(P)$, where $P>0$ is the unique solution to the following Lyapunov equation:

$$
\left[\begin{array}{cc}
-h_{1} & \alpha_{2} \\
\alpha_{3} & \alpha_{4}
\end{array}\right]^{T} P+P\left[\begin{array}{cc}
-h_{1} & \alpha_{2} \\
\alpha_{3} & \alpha_{4}
\end{array}\right]=\left[\begin{array}{cc}
-2 & 0 \\
0 & -2
\end{array}\right] \text {. }
$$

Proof. From (2.1), (2.5) with

$$
e_{i}(t):=x_{i}(t)-\widehat{x}_{i}(t), \quad \forall i \in\{1,2,3,4\},
$$

it can be readily obtained that

$$
\begin{aligned}
e_{4}(t) & =x_{4}(t)-\widehat{x}_{4}(t)=0, \quad \forall t \geq 0, \\
e_{3}(t) & =x_{3}(t)-\widehat{x}_{3}(t) \\
& =x_{3}(t)-\frac{1}{r_{2}}\left[\dot{y}-g_{4}(y)\right] \\
& =x_{3}(t)-\frac{1}{r_{2}}\left[\dot{x}_{4}-g_{4}\left(x_{4}\right)\right] \\
& =x_{3}(t)-\frac{1}{r_{2}}\left[r_{2} x_{3}(t)+g_{4}\left(x_{4}\right)-g_{4}\left(x_{4}\right)\right] \\
& =0, \quad \forall t \geq 0 \\
\dot{e}_{2}(t) & =\dot{x}_{2}(t)-\hat{\dot{x}}_{2}(t) \\
& =\alpha_{3} x_{1}+\alpha_{4} x_{2}+g_{2}\left(x_{4}\right)-\alpha_{3} \widehat{x}_{1}-\alpha_{4} \widehat{x}_{2}-g_{2}\left(\widehat{x}_{4}\right) \\
& =\alpha_{3}\left(x_{1}-\widehat{x}_{1}\right)+\alpha_{4}\left(x_{2}-\widehat{x}_{2}\right)+\left[g_{2}\left(x_{4}\right)-g_{2}\left(\widehat{x}_{4}\right)\right]
\end{aligned}
$$




$$
\begin{aligned}
& =\alpha_{3} e_{1}+\alpha_{4} e_{2}+\left[g_{2}(y)-g_{2}(y)\right] \\
& =\alpha_{3} e_{1}(t)+\alpha_{4} e_{2}(t), \quad \forall t \geq 0 \\
\dot{e}_{1}(t) & =\dot{x}_{1}(t)-\hat{\dot{x}}_{1}(t) \\
& =\alpha_{1} x_{1}+\alpha_{2} x_{2}+g_{1}\left(x_{3}, x_{4}\right)-\frac{\left(\alpha_{1}+h_{1}\right)\left[\dot{x}_{3}-g_{3}(y)\right]}{r_{1} \widehat{x}_{3}}+h_{1} \widehat{x}_{1}-\alpha_{2} \widehat{x}_{2}-g_{1}\left(\widehat{x}_{3}, y\right) \\
& =\alpha_{1} x_{1}+\alpha_{2} e_{2}+g_{1}\left(x_{3}, x_{4}\right)-\frac{\left(\alpha_{1}+h_{1}\right)\left[\dot{x}_{3}-g_{3}\left(x_{4}\right)\right]}{r_{1} x_{3}}+h_{1} \widehat{x}_{1}-g_{1}\left(x_{3}, x_{4}\right) \\
& =\alpha_{1} x_{1}+\alpha_{2} e_{2}-\frac{\left(\alpha_{1}+h_{1}\right)\left[g_{3}\left(x_{4}\right)+r_{1} x_{1} x_{3}-g_{3}\left(x_{4}\right)\right]}{r_{1} x_{3}}+h_{1} \widehat{x}_{1} \\
& =\alpha_{1} x_{1}+\alpha_{2} e_{2}-\left(\alpha_{1}+h_{1}\right) x_{1}+h_{1} \widehat{x}_{1} \\
& =h_{1} e_{1}(t)+\alpha_{2} e_{2}(t), \quad \forall t \geq 0 .
\end{aligned}
$$

This implies that

$$
\left[\begin{array}{c}
\dot{e}_{1}(t) \\
\dot{e}_{2}(t)
\end{array}\right]=\left[\begin{array}{cc}
-h_{1} & \alpha_{2} \\
\alpha_{3} & \alpha_{4}
\end{array}\right]\left[\begin{array}{c}
e_{1}(t) \\
e_{2}(t)
\end{array}\right], \quad\left[\begin{array}{c}
e_{3}(t) \\
e_{4}(t)
\end{array}\right]=0, \quad \forall t \geq 0
$$

with $\sigma\left(\left[\begin{array}{cc}-h_{1} & \alpha_{2} \\ \alpha_{3} & \alpha_{4}\end{array}\right]\right) \subseteq C^{-}$, in view of (A1):

Let

$$
W(t):=\left[\begin{array}{ll}
e_{1}(t) & e_{2}(t)
\end{array}\right] P\left[\begin{array}{l}
e_{1}(t) \\
e_{2}(t)
\end{array}\right]
$$

The time derivative of $W(t)$ along the trajectories of dynamical error system, with (2.9), (2.10), and (2.6), is given by

$$
\begin{aligned}
\dot{W}(t) & =\left[\begin{array}{ll}
\dot{e}_{1} & \dot{e}_{2}
\end{array}\right] P\left[\begin{array}{l}
e_{1} \\
e_{2}
\end{array}\right]+\left[\begin{array}{ll}
e_{1} & e_{2}
\end{array}\right] P\left[\begin{array}{l}
\dot{e}_{1} \\
\dot{e}_{2}
\end{array}\right] \\
& =\left[\begin{array}{ll}
e_{1} & e_{2}
\end{array}\right]\left[\begin{array}{ll}
-h_{1} & \alpha_{2} \\
\alpha_{3} & \alpha_{4}
\end{array}\right]^{T} P\left[\begin{array}{l}
e_{1} \\
e_{2}
\end{array}\right]+\left[\begin{array}{ll}
e_{1} & e_{2}
\end{array}\right] P\left[\begin{array}{ll}
-h_{1} & \alpha_{2} \\
\alpha_{3} & \alpha_{4}
\end{array}\right]\left[\begin{array}{l}
e_{1} \\
e_{2}
\end{array}\right] \\
& =\left[\begin{array}{ll}
e_{1} & e_{2}
\end{array}\right]\left\{\left[\begin{array}{ll}
-h_{1} & \alpha_{2} \\
\alpha_{3} & \alpha_{4}
\end{array}\right]^{T} P+P\left[\begin{array}{ll}
-h_{1} & \alpha_{2} \\
\alpha_{3} & \alpha_{4}
\end{array}\right]\right\}\left[\begin{array}{l}
e_{1} \\
e_{2}
\end{array}\right] \\
& =-2\left[\begin{array}{ll}
e_{1} & e_{2}
\end{array}\right]\left[\begin{array}{l}
e_{1} \\
e_{2}
\end{array}\right] \\
& \leq-\frac{2}{\lambda_{\max }(P)} W(t) \\
& =-2 \alpha W(t), \quad \forall t \geq 0 .
\end{aligned}
$$


Thus, one has

$$
e^{2 \alpha t} \cdot \dot{W}+e^{2 \alpha t} \cdot 2 \alpha W=\frac{d}{d t}\left[e^{2 \alpha t} \cdot W\right] \leq 0, \quad \forall t \geq 0
$$

It follows that

$$
\int_{0}^{t} \frac{d}{d \tau}\left[e^{2 \alpha \tau} \cdot W(\tau)\right] d \tau=e^{2 \alpha t} \cdot W(t)-W(0) \leq \int_{0}^{t} 0 d \tau=0
$$

Consequently, we conclude that

$$
\begin{aligned}
\|e(t)\| & =\sqrt{e_{1}^{2}(t)+e_{2}^{2}(t)+e_{3}^{2}(t)+e_{4}^{2}(t)} \\
& =\sqrt{e_{1}^{2}(t)+e_{2}^{2}(t)} \\
& \leq \sqrt{\frac{W(t)}{\lambda_{\min }(P)}} \\
& \leq \sqrt{\frac{e^{-2 \alpha t} W(0)}{\lambda_{\min }(P)}} \\
& =\sqrt{\frac{W(0)}{\lambda_{\min }(P)}} \cdot e^{-\alpha t}, \quad \forall t \geq 0,
\end{aligned}
$$

in view of (2.8), (2.10), and (2.13). This completes the proof.

\section{Numerical Simulations}

Consider the generalized hyperchaotic system:

$$
\begin{gathered}
\dot{x}_{1}(t)=-x_{2}-x_{3}, \\
\dot{x}_{2}(t)=a x_{1}+b x_{2}+x_{4}, \\
\dot{x}_{3}(t)=x_{1} x_{3}+3, \\
\dot{x}_{4}(t)=-0.5 x_{3}+0.05 x_{4}, \\
y(t)=x_{4} .
\end{gathered}
$$


Case 1 ( $(a=1, b=0.25)$ or, equivalently, the Rössler hyperchaotic system). It can be verified that condition (A1) is satisfied with $h_{1}=1.2$. By Theorem 2.3, we conclude that system (3.1) with $a=1$ and $b=0.25$ is exponentially state reconstructible by the nonlinear observer:

$$
\begin{gathered}
\widehat{x}_{3} \dot{\hat{x}}_{1}=1.2\left(\dot{\hat{x}}_{3}-3\right)-1.2 \widehat{x}_{1} \widehat{x}_{3}-\widehat{x}_{2} \widehat{x}_{3}-\widehat{x}_{3}^{2}, \\
\dot{\hat{x}}_{2}=\widehat{x}_{1}+0.25 \widehat{x}_{2}+y, \\
\hat{x}_{3}=0.1 y-2 \dot{y}, \\
\hat{x}_{4}=y
\end{gathered}
$$

with the guaranteed exponential decay rate $\alpha=0.164$.

Case $2(a=-20, b=-50)$. It can be verified that condition (A1) is satisfied with $h_{1}=10$. By Theorem 2.3, we conclude that system (3.1) with $a=-20$ and $b=-50$ is exponentially state reconstructible by the nonlinear observer:

$$
\begin{gathered}
\widehat{x}_{3} \dot{\hat{x}}_{1}=10\left(\dot{\hat{x}}_{3}-3\right)-10 \widehat{x}_{1} \widehat{x}_{3}-\widehat{x}_{2} \widehat{x}_{3}-\widehat{x}_{3}^{2}, \\
\dot{\hat{x}}_{2}=-20 \widehat{x}_{1}-50 \widehat{x}_{2}+y, \\
\widehat{x}_{3}=0.1 y-2 \dot{y}, \\
\widehat{x}_{4}=y,
\end{gathered}
$$

with the guaranteed exponential decay rate $\alpha=8.47$.

Case 3 ( $a=30, b=-40)$. It can be verified that condition (A1) is satisfied with $h_{1}=5$. By Theorem 2.3, we conclude that system (3.1) with $a=30$ and $b=-40$ is exponentially state reconstructible by the nonlinear observer:

$$
\begin{gathered}
\widehat{x}_{3} \dot{\hat{x}}_{1}=5\left(\dot{\hat{x}}_{3}-3\right)-5 \widehat{x}_{1} \widehat{x}_{3}-\widehat{x}_{2} \widehat{x}_{3}-\widehat{x}_{3}^{2}, \\
\dot{\hat{x}}_{2}=30 \widehat{x}_{1}-40 \widehat{x}_{2}+y, \\
\widehat{x}_{3}=0.1 y-2 \dot{y}, \\
\hat{x}_{4}=y,
\end{gathered}
$$

with the guaranteed exponential decay rate $\alpha=3.79$.

The time response of error states for system (3.1) with Case 1-Case 3 is depicted in Figures 1, 2, and 3, respectively. From the foregoing simulations results, it is seen that system (3.1) with Case 1-Case 3, regardless of chaotic system or nonchaotic system, is exponentially state reconstructible by the nonlinear observers (3.2)-(3.4), respectively. 


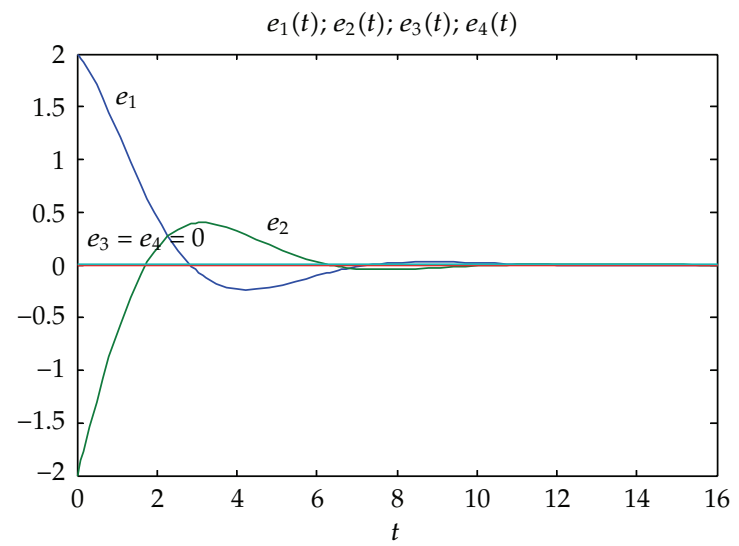

Figure 1: The time response of error states, with $a=1$ and $b=0.25$.

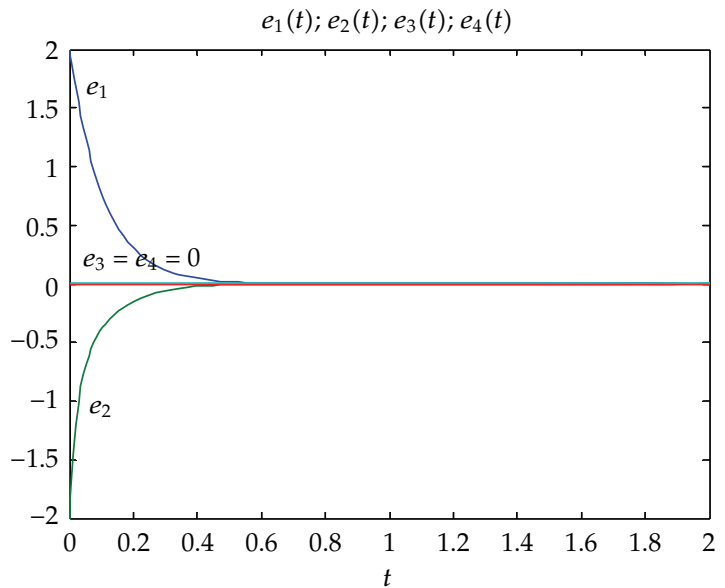

Figure 2: The time response of error states, with $a=-20$ and $b=-50$.

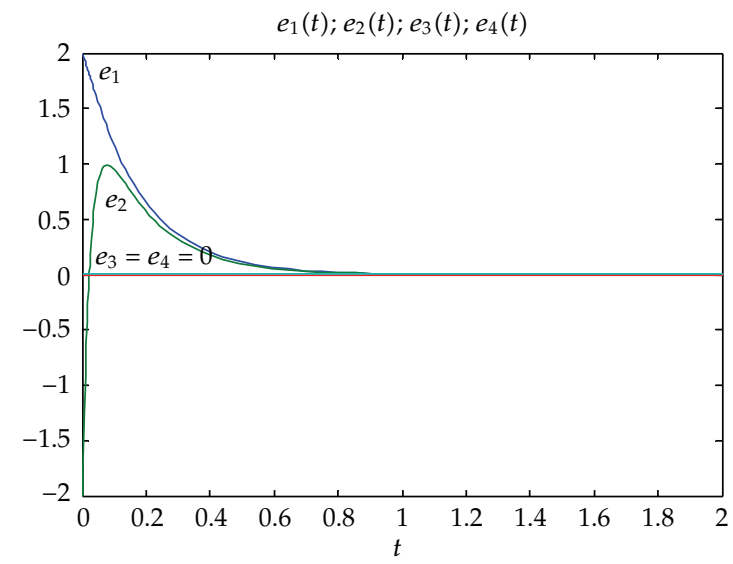

Figure 3: The time response of error states, with $a=30$ and $b=-40$. 


\section{Conclusion}

In this paper, the generalized Rössler hyperchaotic systems have been presented, and the state observation problem of such systems has been investigated. Based on the DIL methodology, a nonlinear state reconstructor of the generalized Rössler hyperchaotic systems has been developed to guarantee the global exponential stability of the resulting error system. Besides, the guaranteed exponential decay rate can be accurately estimated. However, the state observation design for more general uncertain hyperchaotic system still remains unanswered. This constitutes an interesting future research problem.

\section{Nomenclature}

$\Re^{n}: \quad$ The $n$-dimensional real space

$C^{-}: \quad$ The set of $\{a+b j \mid a<0, b \in \Re\}$

$|a|: \quad$ The modulus of a real number $a$

$\|x\|: \quad$ The Euclidean norm of the vector $x \in \mathfrak{R}^{n}$

$\|A\|: \quad$ The induced Euclidean norm of the matrix $A$

$A^{T}$ : $\quad$ The transpose of the matrix $A$

$\sigma(A)$ : The set of all eigenvalues of the matrix $A$

$P>0$ : $\quad$ The symmetric matrix $P$ is positive definite

$\lambda_{\max }(P)$ : The maximum eigenvalue of the symmetric matrix $P$ with real eigenvalues

$\lambda_{\min }(P)$ : The minimum eigenvalue of the symmetric matrix $P$ with real eigenvalues.

\section{Acknowledgments}

The author thanks the National Science Council of Republic of China for supporting this work under Grant NSC-100-2221-E-214-015. The author also wishes to thank the anonymous reviewers for providing constructive suggestions.

\section{References}

[1] J. Baek, H. Lee, S. Kim, and M. Park, "Adaptive fuzzy bilinear observer based synchronization design for generalized Lorenz system," Physics Letters A, vol. 373, no. 47, pp. 4368-4375, 2009.

[2] D. Ghosh, P. Saha, and A. R. Chowdhury, "Linear observer based projective synchronization in delay Rössler system," Communications in Nonlinear Science and Numerical Simulation, vol. 15, no. 6, pp. 16401647, 2010.

[3] B. Jovic and C. P. Unsworth, "Fast synchronisation of chaotic maps for secure chaotic communications," Electronics Letters, vol. 46, no. 1, pp. 49-50, 2010.

[4] H. K. Lam, "Chaotic synchronisation using output/full state-feedback polynomial controller," IET Control Theory \& Applications, vol. 4, no. 11, pp. 2285-2292, 2010.

[5] J. Li, W. Li, and Q. Li, "Sliding mode control for uncertain chaotic systems with input nonlinearity," Communications in Nonlinear Science and Numerical Simulation, vol. 17, no. 1, pp. 341-348, 2012.

[6] Y. Li, "Some new less conservative criteria for impulsive synchronization of a hyperchaotic Lorenz system based on small impulsive signals," Nonlinear Analysis. Real World Applications, vol. 11, no. 2, pp. 713-719, 2010.

[7] J.S. Lin, T. L. Liao, J. J. Yan, and H. T. Yau, "Synchronization of unidirectional coupled chaotic systems with unknown channel time-delay: adaptive robust observer-based approach," Chaos, Solitons and Fractals, vol. 26, no. 3, pp. 971-978, 2005.

[8] Y. J. Sun, "A simple observer design of the generalized Lorenz chaotic systems," Physics Letters A, vol. 374, no. 7, pp. 933-937, 2010.

[9] C. S. Ting, "An adaptive fuzzy observer-based approach for chaotic synchronization," International Journal of Approximate Reasoning, vol. 39, no. 1, pp. 97-114, 2005. 
[10] H. T. Yau, J. S. Lin, and J. J. Yan, "Synchronization control for a class of chaotic systems with uncertainties," International Journal of Bifurcation and Chaos in Applied Sciences and Engineering, vol. 15, no. 7, pp. 2235-2246, 2005.

[11] F. Zhu, "Observer-based synchronization of uncertain chaotic system and its application to secure communications," Chaos, Solitons and Fractals, vol. 40, no. 5, pp. 2384-2391, 2009.

[12] B. Chen, J. Lam, and Z. Wang, "Observer design and stabilization for linear neutral delay systems," ISA Transactions, vol. 44, no. 1, pp. 35-42, 2005.

[13] Z. G. Li, C. Y. Wen, and Y. C. Soh, "Observer-based stabilization of switching linear systems," Automatica, vol. 39, no. 3, pp. 517-524, 2003.

[14] L. A. Torres, V. Ibarra-Junquera, P. Escalante-Minakata, and H. C. Rosu, "High-gain nonlinear observer for simple genetic regulation process," Physica A, vol. 380, no. 1-2, pp. 235-240, 2007.

[15] R. Engel and G. Kreisselmeier, "Nonlinear approximate observers for feedback control," Systems and Control Letters, vol. 56, no. 3, pp. 230-235, 2007.

[16] M. Hajatipour and M. Farrokhi, "Chattering free with noise reduction in sliding-mode observers using frequency domain analysis," Journal of Process Control, vol. 20, no. 8, pp. 912-921, 2010.

[17] F. A. Shaik, S. Purwar, and B. Pratap, "Real-time implementation of Chebyshev neural network observer for twin rotor control system," Expert Systems with Applications, vol. 38, no. 10, pp. 1304313049, 2011.

[18] H. Shim, J. H. Seo, and A. R. Teel, "Nonlinear observer design via passivation of error dynamics," Automatica, vol. 39, no. 5, pp. 885-892, 2003.

[19] H. Trinh, M. Aldeen, and S. Nahavandi, "An observer design procedure for a class of nonlinear timedelay systems," Computers and Electrical Engineering, vol. 30, no. 1, pp. 61-71, 2004.

[20] Y. Wang and A. F. Lynch, "Observer design using a generalized time-scaled block triangular observer form," Systems and Control Letters, vol. 58, no. 5, pp. 346-352, 2009.

[21] M. Al-Sawalha Mossa and M. S. M. Noorani, "Application of the differential transformation method for the solution of the hyperchaotic Rössler system," Communications in Nonlinear Science and Numerical Simulation, vol. 14, no. 4, pp. 1509-1514, 2009. 


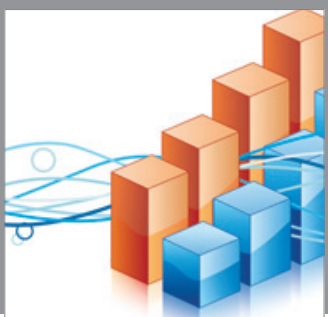

Advances in

Operations Research

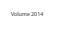

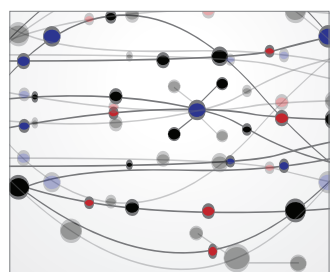

\section{The Scientific} World Journal
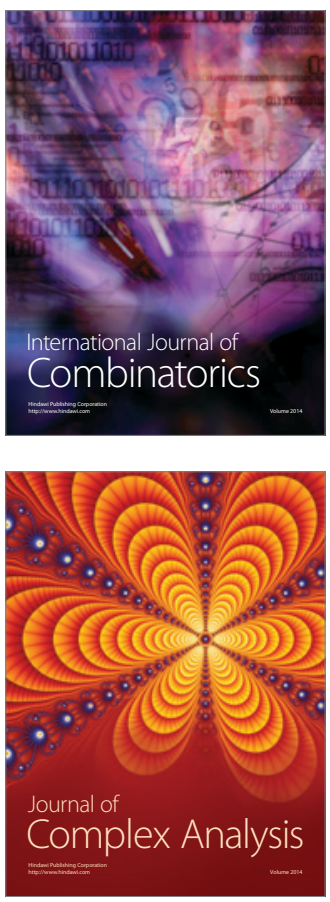

International Journal of

Mathematics and

Mathematical

Sciences
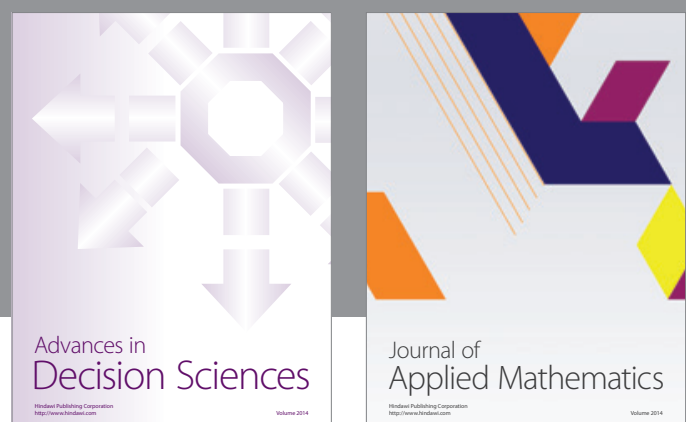

Journal of

Applied Mathematics
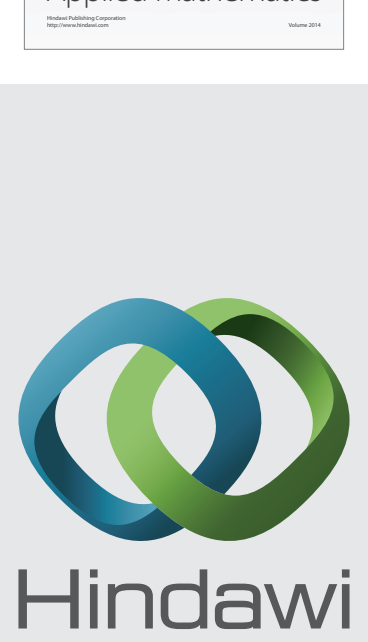

Submit your manuscripts at http://www.hindawi.com
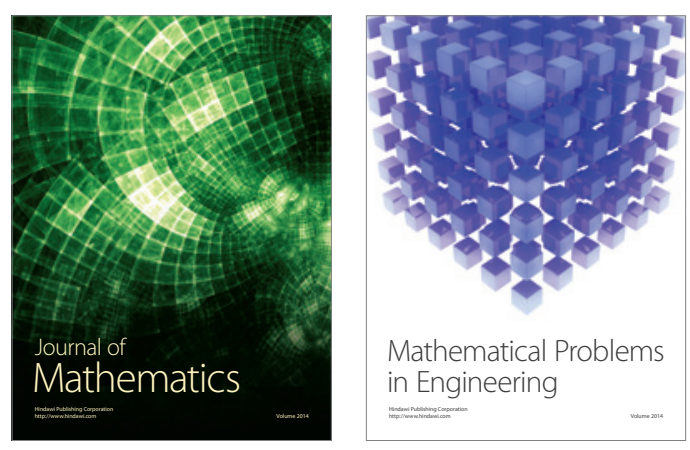

Mathematical Problems in Engineering
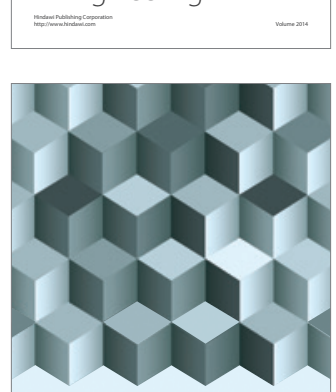

Journal of

Function Spaces
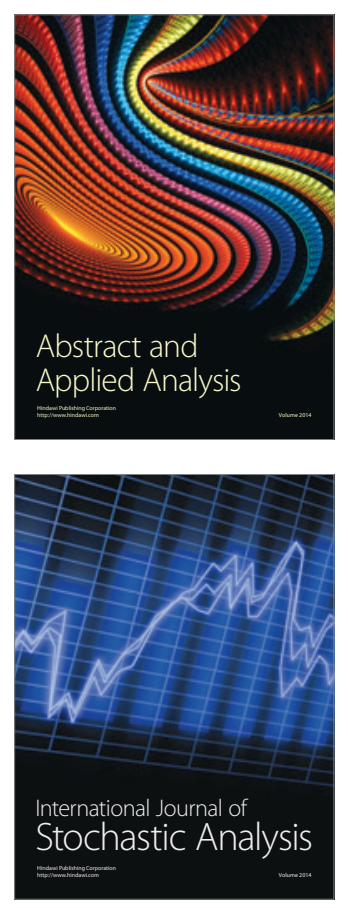

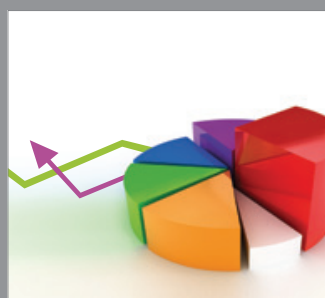

ournal of

Probability and Statistics

Promensencen
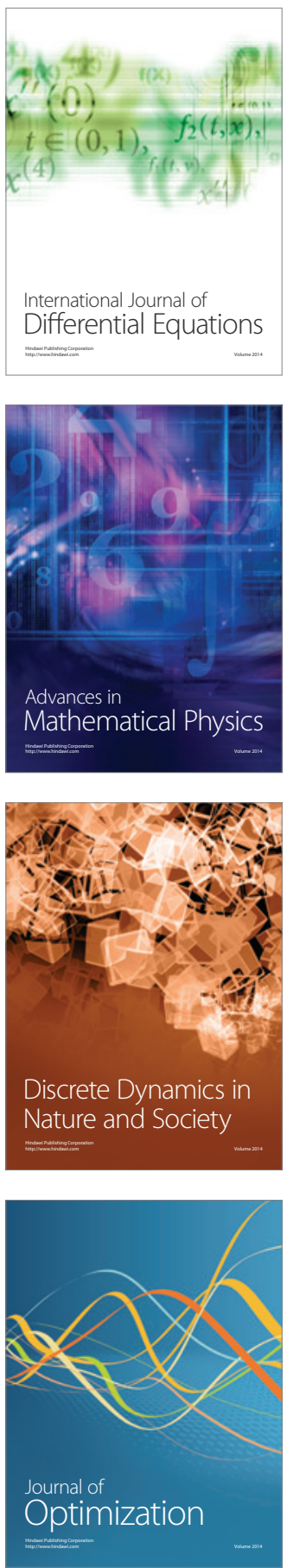